\title{
Addressing the Feasibility of the Parameters of Strongly Regular Graphs with a MacLaurin Series Approach
}

\author{
Vasco Moco Mano, Lu1s Vieira \\ Department of Civil Engineering of Faculty of Engineering of University of Porto, Portugal
}

\begin{abstract}
In the environment of Euclidean Jordan algebras, we consider a Jordan frame associated to the adjacency matrix of a strongly regular graph and construct a MacLaurin series with an element of the frame. From this series we establish feasibility conditions for the existence of strongly regular graphs.
\end{abstract}

Keywords-Strongly regular graph, Euclidean Jordan algebra, Matrix analysis.

\section{INTRODUCTION}

$\mathbf{O}$ $\mathrm{N}$ the article Strongly regular graphs, partial geometries and partially balanced designs, [1], R. C. Bose introduced a class of regular graphs governed by a set of four parameters. One very challenging problem concerning these graphs, lately called strongly regular, is to find good feasibility conditions over their parameter sets, in order to filter potential strongly regular graphs. A short survey on the theory of strongly regular graphs is made in Section II.

Throughout this paper we take an Euclidean Jordan algebraic approach to address this problem. By considering a special Euclidean Jordan algebra associated to the adjacency matrix of a strongly regular graph, Section IV, we constructed a MacLaurin series that allowed us to establish some feasibility conditions over the set of parameters of a strongly regular graph, Section V.

Euclidean Jordan algebras were introduced in the paper [2] and, since then, they have been applied to different branches of mathematics. For instance, there are applications to statistics, [3], to interior point methods, [4] or [5] and to combinatorics, [6], [7], [8]. The basic definitions and main results for our work are presented in Section III.

We finish our paper with some experimental results where we test our admissibility conditions as well as some conclusions, Section VI.

\section{General Concepts on Strongly Regular GRAPHS}

Along this paper, we consider only simple graphs, that is, graphs without loops and parallel edges, herein called graphs.

A graph in which all pairs of vertices are adjacent (nonadjacent) is called a complete (null) graph. The number of neighbors of a vertex $v$ in $V(X)$ is called the degree of $v$.
If all vertices of a graph $X$ have degree $k$, for some natural number $k$, then $X$ is $k$-regular.

We associate to a graph $X$ an $n$ by $n$ matrix $A=\left[a_{i j}\right]$, where each $a_{i j}=1$, if $v_{i} v_{j} \in E(X)$, otherwise $a_{i j}=0$, called the adjacency matrix of $X$. The eigenvalues of $A$ are simply called the eigenvalues of $X$.

A non-null and not complete graph $X$ is strongly regular with parameters $(n, k, a, c)$ if it is $k$-regular, each pair of adjacent vertices has $a$ common neighbors and each pair of non-adjacent vertices have $c$ common neighbors. It is well known that the parameter set, $(n, k, a, c)$, of a strongly regular graph satisfy the following equality

$$
k(k-a-1)=(n-k-1) c .
$$

Also (see, for instance, [9]), the eigenvalues of a strongly regular graph $X$ with parameters $(n, k, a, c)$ are $k, \theta$ and $\tau$, where $\theta$ and $\tau$ are given by

$$
\begin{aligned}
\theta & =\frac{a-c+\sqrt{(a-c)^{2}+4(k-c)}}{2} \\
\tau & =\frac{a-c-\sqrt{(a-c)^{2}+4(k-c)}}{2} .
\end{aligned}
$$

Note that $\theta$ and $\tau$, usually called the restricted eigenvalues of $X$, are such that the former is positive and the latter is negative. Their multiplicities can also be obtained from the parameters of $X$ as follows (see, for instance, [9]):

$$
\begin{aligned}
& m_{\theta}=\frac{1}{2}\left(n-1-\frac{(\theta+\tau)(n-1)+2 k}{\theta-\tau}\right) \\
& m_{\tau}=\frac{1}{2}\left(n-1+\frac{(\theta+\tau)(n-1)+2 k}{\theta-\tau}\right),
\end{aligned}
$$

where $m_{\theta}$ and $m_{\tau}$ are the multiplicities of $\theta$ and $\tau$, respectively.

If $X$ is a strongly regular graph with parameters $(n, k, a, c)$, then its complement graph, $\bar{G}$ is also strongly regular with parameters $(n, \bar{k}, \bar{a}, \bar{c})$, where

$$
\begin{aligned}
\bar{k} & =n-k-1, \\
\bar{a} & =n-2-2 k+c, \\
\bar{c} & =n-2 k+a .
\end{aligned}
$$

A very important problem on the study of these graphs is to find suitable admissibility conditions over their parameter sets in order to allow us to decide whether a given parameter set can produce a strongly regular graph or not. We have already presented some trivial feasibility conditions for the parameters 
of a strongly regular graph. In fact, it is clear that given any natural numbers $k, a$ and $c$, equality (1) has to produce a natural $n$. Using a same kind of reasoning, formulas (4) and (5) must produce natural numbers for $m_{\theta}$ and $m_{\tau}$. Finally, note that while (6) and (8) yield positive numbers, the same is not guaranteed for (7). Besides these trivial feasibility conditions, there are many nontrivial conditions widely used for checking the feasibility of a parameter set of a strongly regular graph.

The eigenvalues of a strongly regular graph satisfy the following inequalities, known as the Krein conditions, [11]:

$$
\begin{aligned}
& (\theta+1)(k+\theta+2 \theta \tau) \leq(k+\theta)(\tau+1)^{2}, \\
& (\tau+1)(k+\tau+2 \theta \tau) \leq(k+\tau)(\theta+1)^{2} .
\end{aligned}
$$

It was also proved (see [12]) that the multiplicities of the eigenvalues satisfy the so-called Seidel's absolute bounds:

$$
\begin{aligned}
& n \leq \frac{m_{\theta}\left(m_{\theta}+3\right)}{2}, \\
& n \leq \frac{m_{\tau}\left(m_{\tau}+3\right)}{2} .
\end{aligned}
$$

These inequalities where improved by Neumaier (see [13]) in some particular cases.

Finally, A. E. Brouwer obtained the following condition (see [14]), known as the claw bound:

$$
2(\theta+1) \leq \tau(\tau+1)(c+1)
$$

if $c \neq \tau^{2}$ and $c \neq \tau(\tau+1)$.

We say that a parameter set $(n, k, a, c)$ is feasible if all of the above conditions are satisfied. With these conditions, many of the parameter sets are discarded as possible parameters sets of strongly regular graphs. However, to decide whether a set of parameters is the parameter set of a strongly regular graph it is one of the main problems on the study of strongly regular graphs. In fact, there are still many parameter sets for which we do not know if they correspond to a strongly regular graph. To address some of these open problems we studied the relationship between these graphs and Euclidean Jordan algebras, which will be presented in Section III.

It is worth notice that the Krein conditions (9)-(10) and the Seidel's absolute bounds (11)-(12) are special cases of general inequalities obtained for association schemes, since strongly regular graphs are symmetric association schemes with two classes. For more information on these more complex combinatorial structures it is suggested the reading of [10], for instance.

\section{A SHORT INTRODUCTION TO EUCLIDEAN JORDAN ALGEBRAS}

In this section relevant concepts for our work are shortly surveyed. These can be seen, for instance, in [15].

Let $\mathcal{V}$ be a real vector space with finite dimension and a bilinear mapping $(u, v) \mapsto u \bullet v$ from $\mathcal{V} \times \mathcal{V}$ to $\mathcal{V}$, such that for each $u \in \mathcal{V}$ the algebra spanned by $u$ is associative. Then, $\mathcal{V}$ is called a real power associative algebra. If $\mathcal{V}$ contains an element, $e$, such that for all $u$ in $\mathcal{V}, e \bullet u=u \bullet e=u$, then $e$ is called the unit element of $\mathcal{V}$.
Considering a bilinear mapping $(u, v) \mapsto u \bullet v$, if for all $u$ and $v$ in $\mathcal{V}$ we have $\left(J_{1}\right) u \bullet v=v \bullet u$ and $\left(J_{2}\right) u \bullet\left(u^{2} \bullet v\right)=$ $u^{2} \bullet(u \bullet v)$, with $u^{2}=u \bullet u$, then $\mathcal{V}$ is called a Jordan algebra. If $\mathcal{V}$ is a Jordan algebra with unit element, then $\mathcal{V}$ is power associative (see [15]). Along this paper we only consider finite dimensional real Jordan algebras.

Given a Jordan algebra $\mathcal{V}$ with unit element $e$, if there is an inner product $\langle\cdot, \cdot\rangle$ that verifies the equality $\langle u \bullet v, w\rangle=<$ $v, u \bullet w>$, for any $u, v, w$ in $\mathcal{V}$, then $\mathcal{V}$ is called an Euclidean Jordan algebra.

An element $c$ in an Euclidean Jordan algebra $\mathcal{V}$, with unit element $e$, is an idempotent if $c^{2}=c$. Two idempotents $c$ and $d$ are orthogonal if $c \bullet d=0$. An idempotent is primitive if it is non-zero and cannot be written as the sum of two non-zero idempotents.

We call the set $\left\{c_{1}, c_{2}, \ldots, c_{k}\right\}$ a complete system of orthogonal idempotents if

(i) $c_{i}^{2}=c_{i}, \forall i \in\{1, \ldots, k\}$;

(ii) $c_{i} \bullet c_{j}=0, \forall i \neq j$ and

(iii) $c_{1}+c_{2}+\cdots+c_{k}=e$

Additionally, if every $c_{i}, i \in\{1, \ldots, k\}$ is primitive, $\left\{c_{1}, c_{2}, \ldots, c_{k}\right\}$ is called a Jordan frame.

Let $\mathcal{V}$ be an Euclidean Jordan algebra with unit element $e$. Then, for every $u$ in $\mathcal{V}$, there are unique distinct real numbers $\lambda_{1}, \lambda_{2}, \ldots, \lambda_{k}$, and an unique complete system of orthogonal idempotents $\left\{c_{1}, c_{2}, \ldots, c_{k}\right\}$ such that

$$
u=\lambda_{1} c_{1}+\lambda_{2} c_{2}+\cdots+\lambda_{k} c_{k},
$$

with $c_{j} \in \mathbb{R}[u], j=1, \ldots, k$, see [15, Theorem III.1.1]. These $\lambda_{j}$ 's are the eigenvalues of $u$ and (14) is called the first spectral decomposition of $u$. A similar result for Jordan frames is given in [15, Theorem III.1.2] and we obtain the second spectral decomposition that is analogous to (14). The difference is that in the second spectral decomposition the $\lambda$ 's are not all distinct and appear with their respective multiplicities.

The rank of an element $u$ in $\mathcal{V}$ is the least natural number $k$, such that the set $\left\{e, u, \ldots, u^{k}\right\}$ is linearly dependent (where $\left.u^{k}=u \bullet u^{k-1}\right)$, and we write $\operatorname{rank}(u)=k$. This concept is expanded by defining the rank of the algebra $\mathcal{V}$ as the natural number $\operatorname{rank}(\mathcal{V})=\max \{\operatorname{rank}(u): u \in \mathcal{V}\}$. The elements of $\mathcal{V}$ with rank equal to the rank of $\mathcal{V}$ are the regular elements of $\mathcal{V}$. This set of the regular elements is an open and dense subset in $\mathcal{V}$. If $u$ is a regular element of $\mathcal{V}$, with $r=\operatorname{rank}(u)$, then the set $\left\{e, u, u^{2}, \ldots, u^{r}\right\}$ is linearly dependent and the set $\left\{e, u, u^{2}, \ldots, u^{r-1}\right\}$ is linearly independent. Thus we may conclude that there exist unique real numbers $a_{1}(u), \ldots, a_{r}(u)$, such that $u^{r}-a_{1}(u) u^{r-1}+$ $\cdots+(-1)^{r} a_{r}(u) e=0$, where 0 is the null vector of $\mathcal{V}$. Making the necessary adjustments we obtain the polynomial in $\lambda$

$$
p(u, \lambda)=\lambda^{r}-a_{1}(u) \lambda^{r-1}+\cdots+(-1)^{r} a_{r}(u),
$$

that is called the characteristic polynomial of $u$, where each coefficient $a_{i}$ is a homogeneous polynomial of degree $i$ in the coordinates of $u$ in a fixed basis of $\mathcal{V}$. Although the characteristic polynomial is defined for a regular element of $\mathcal{V}$, we can extend this definition to all the elements of $\mathcal{V}$, since each polynomial $a_{i}$ is homogeneous and the set of regular 
elements of $\mathcal{V}$ is dense in $\mathcal{V}$. The roots of the characteristic polynomial of $u, \lambda_{1}, \lambda_{2}, \ldots, \lambda_{r}$, are called the eigenvalues of $u$. Furthermore, the coefficients $a_{1}(u)$ and $a_{r}(u)$ of the characteristic polynomial of $u$, are called the trace and the determinant of $u$, respectively.

\section{A Jordan Frame Associated to A Strongly REGULAR GRAPH}

We consider a strongly regular graph in the environment of Euclidean Jordan algebras, in a similar way as we did in [8].

From now on we consider the Euclidean Jordan algebra of real symmetric matrices of order $n, \mathcal{V}$, such that $\forall A, B \in \mathcal{V}$, $A \bullet B=(A B+B A) / 2$, where $A B$ is the usual product of matrices. Furthermore, the inner product of $\mathcal{V}$ is defined as $<A, B>=\operatorname{tr}(A B)$, where $\operatorname{tr}(\cdot)$ denotes the classical trace of matrices, that is the sum of its eigenvalues.

Let $X$ be a strongly regular graph with parameter set $(n, k, a, c)$ and let $A$ be the adjacency matrix of $X$ with three distinct eigenvalues, namely the degree of regularity $k$, and the restricted eigenvalues $\theta$ and $\tau$, given in (2) and (3). Now we consider the Euclidean Jordan subalgebra of $\mathcal{V}, \mathcal{V}^{\prime}$, spanned by the identity matrix of order $n, I_{n}$, and the powers of $A$. Since $A$ has three distinct eigenvalues, then $\mathcal{V}^{\prime}$ is a three dimensional Euclidean Jordan algebra with $\operatorname{rank}\left(\mathcal{V}^{\prime}\right)=3$.

Let $\mathcal{B}=\left\{E_{0}, E_{1}, E_{2}\right\}$ be the Jordan frame of $\mathcal{V}^{\prime}$ associated to $A$, with

$$
\begin{aligned}
& E_{0}=\frac{A^{2}-(\theta+\tau) A+\theta \tau I_{n}}{(k-\theta)(k-\tau)}=\frac{J_{n}}{n}, \\
& E_{1}=\frac{A^{2}-(k+\tau) A+k \tau I_{n}}{(\theta-\tau)(\theta-k)}, \\
& E_{2}=\frac{A^{2}-(k+\theta) A+k \theta I_{n}}{(\tau-\theta)(\tau-k)},
\end{aligned}
$$

where $J_{n}$ is the matrix whose entries are all equal to 1 . The elements of $\mathcal{B}$ can be rewritten under the basis $\left\{I_{n}, A, J_{n}-\right.$ $\left.A-I_{n}\right\}$ of $\mathcal{V}^{\prime}$ in the following manner:

$$
\begin{aligned}
E_{0} & =\frac{\theta-\tau}{n(\theta-\tau)} I_{n}+\frac{\theta-\tau}{n(\theta-\tau)} A \\
& +\frac{\theta-\tau}{n(\theta-\tau)}\left(J_{n}-A-I_{n}\right) \\
E_{1} & =\frac{|\tau| n+\tau-k}{n(\theta-\tau)} I_{n}+\frac{n+\tau-k}{n(\theta-\tau)} A \\
& +\frac{\tau-k}{n(\theta-\tau)}\left(J_{n}-A-I_{n}\right), \\
E_{2} & =\frac{\theta n+k-\theta}{n(\theta-\tau)} I_{n}+\frac{-n+k-\theta}{n(\theta-\tau)} A \\
& +\frac{k-\theta}{n(\theta-\tau)}\left(J_{n}-A-I_{n}\right) .
\end{aligned}
$$

Let $\mathcal{M}_{n}(\mathbb{R})$ the space of square matrices with real entries and by $\mathcal{M}_{m, n}(\mathbb{R})$ the space of $m \times n$ matrices with real entries. We consider the Hadamard product, defined for two matrices $A, B$ of order $n$ as the componentwise product: $(A \circ B)_{i j}=$ $A_{i j} B_{i j}$ and the Kronecker product, for matrices $C=\left[c_{i j}\right] \in$
$\mathcal{M}_{m, n}(\mathbb{R})$ and $D=\left[d_{i j}\right] \in \mathcal{M}_{p, q}(\mathbb{R})$, defined by

$$
C \otimes D=\left(\begin{array}{ccc}
c_{11} D & \cdots & c_{1 n} D \\
\vdots & \ddots & \vdots \\
c_{m 1} D & \cdots & c_{m n} D
\end{array}\right) .
$$

We now introduce a specific notation that will be used in the following sections. Consider the natural number $p$. Then, for $A \in M_{n}(\mathbb{R})$, we denote by $A^{\circ p}$ and $A^{\otimes p}$ the Hadamard power of order $p$ of $A$ and the Kronecker power of order $p$ of $A$, respectively, with $A^{\circ 1}=A$ and $A^{\otimes 1}=A$.

\section{A Feasibility Condition Associated to A MACLAURIN SERIES}

Consider the idempotent $E_{2}$ given in the previous section. The eigenvalues of $E_{2}$ are given by

$$
\begin{aligned}
q_{0} & =\frac{\theta n+k-\theta}{n(\theta-\tau)}+\frac{-n+k-\theta}{n(\theta-\tau)} k \\
& +\frac{k-\theta}{n(\theta-\tau)}(n-k-1), \\
q_{1} & =\frac{\theta n+k-\theta}{n(\theta-\tau)}+\frac{-n+k-\theta}{n(\theta-\tau)} \theta \\
& +\frac{k-\theta}{n(\theta-\tau)}(-\theta-1), \\
q_{2} & =\frac{\theta n+k-\theta}{n(\theta-\tau)}+\frac{-n+k-\theta}{n(\theta-\tau)} \tau \\
& +\frac{k-\theta}{n(\theta-\tau)}(-\tau-1) .
\end{aligned}
$$

From $E_{2}$ we build the following partial sum:

$$
\begin{aligned}
S_{2 l} & =\sum_{j=1}^{2 l}(-1)^{j-1} \frac{\left(E_{2}^{\circ 2}\right)^{\circ(2 j-1)}}{2 j-1} \\
& +\frac{1}{3}\left(\frac{\theta n+k-\theta}{n(\theta-\tau)}\right)^{3} \frac{1-\left(\frac{\theta n+k-\theta}{n(\theta-\tau)}\right)^{l}}{1-\left(\frac{\theta n+k-\theta}{n(\theta-\tau)}\right)^{4}} I_{n} .
\end{aligned}
$$

Since $\mathcal{V}^{\prime}$ is closed under the Hadamard product and $\mathcal{B}$ is a basis of $\mathcal{V}^{\prime}$, we can write $S_{2 l}$ as:

$$
S_{2 l}=\sum_{i=0}^{2} q_{S_{2 l}}^{i} E_{i},
$$

where the $q_{S_{2 l}}^{i}, i \in\{0,1,2\}$ are the eigenvalues of $S_{2 l}$. We prove that $q_{S_{2 l}}^{i} \geq 0, \forall i \in\{0,1,2\}$. First, we note the following identity regarding one of the eigenvalues of $E_{2}^{\circ 2}$ :

$$
\begin{gathered}
\left(\frac{\theta n+k-\theta}{n(\theta-\tau)}\right)^{2}+\left(\frac{-n+k-\theta}{n(\theta-\tau)}\right)^{2} k \\
+\left(\frac{(k-\theta)}{n(\theta-\tau)}\right)^{2}(n-k-1)=\frac{\theta n+k-\theta}{n(\theta-\tau)} .
\end{gathered}
$$

Secondly, since all of the eigenvalues of $E_{2}$ are smaller in modulus that $q_{0}$, then the summands of $S_{2 l}$, when $j-1$ is odd are smaller, in modulus, than

$$
\left(\frac{\theta n+k-\theta}{n(\theta-\tau)}\right)^{2 j-1} \text {. }
$$


For this assertion we also use the property

$$
\lambda_{\max }\left(\left(A_{1} \circ \ldots \circ A_{i}\right)\right) \leq \lambda_{\max }\left(\left(A_{1}\right)\right) \ldots \lambda_{\max }\left(\left(A_{i}\right)\right),
$$

where $\lambda_{\max }(A)$ denotes the maximum eigenvalue of the matrix $A$. Therefore, we conclude that all the eigenvalues of $S_{2 l}$ are nonnegative.

Now we consider the series:

$$
\begin{aligned}
S_{\infty} & =\left[\arctan \left(\frac{(\theta n+k-\theta)}{n(\theta-\tau)}\right)^{2}\right. \\
& \left.+\frac{1}{3}\left(\frac{\theta n+k-\theta}{n(\theta-\tau)}\right)^{3} \frac{1}{1-\left(\frac{\theta * n+k-\theta}{n(\theta-\tau)}\right)^{4}}\right] I_{n} \\
& +\arctan \left(\frac{-n+k-\theta}{n(\theta-\tau)}\right)^{2} A \\
& +\arctan \left(\frac{k-\theta}{n(\theta-\tau)}\right)^{2}\left(J_{n}-A-I_{n}\right) .
\end{aligned}
$$

Let $q_{\infty}^{i}, i \in\{0,1,2\}$ be the eigenvalues of $S_{\infty}$ such that

$$
S_{\infty}=\sum_{i=0}^{2} q_{\infty}^{i} E_{i}
$$

Then, since $q_{\infty}^{i}=\lim _{l \rightarrow} q_{S_{2 l}}^{i}$, for $i \in\{0,1,2\}$, and $q_{S_{2 l}}^{i} \geq 0$, $\forall i \in\{0,1,2\}$, we conclude that $q_{\infty}^{i} \geq 0, \forall i \in\{0,1,2\}$.

Finally, we consider the new matrix, $S_{2 \infty}$, obtained as

$$
S_{2 \infty}=E_{2} \circ S_{\infty}
$$

The eigenvalues of $S_{2 \infty}$ are also nonnegative because of the non-negativity of the eigenvalues of $E_{2}$ and $S_{\infty}$ and the property $\lambda_{\min }(A \circ B) \geq \lambda_{\min }(A) \lambda_{\min }(B)$, where $\lambda_{\min }(A)$ denotes the minimum eigenvalue of the matrix $A$. From the non-negativity of the eigenvalues of $S_{2 \infty}$ we establish the following result.

Theorem 1: Let $X$ be a strongly regular graph with parameter set $(n, k, a, c)$ and three distinct eigenvalues, $k, \theta$ and $\tau$. If $k<n / 3$ and $\theta<|\tau|-2 / 3$, then

$$
k \leq \frac{1}{3}(3 \theta+1)^{3} .
$$

Proof: Let $q_{2 \infty}^{i}, i \in\{0,1,2\}$ be the eigenvalues of $S_{2 \infty}$ such that

$$
S_{2 \infty}=\sum_{i=0}^{2} q_{2 \infty}^{i} E_{i}
$$

We have already proved that all the eigenvalues of $S_{2 \infty}$ are nonnegative. In particular, we have that $q_{2 \infty}^{0} \geq 0$, that is

$$
\begin{aligned}
0 & \leq \frac{\theta n+k-\theta}{n(\theta-\tau)}\left[\arctan \left(\frac{(\theta n+k-\theta)}{n(\theta-\tau)}\right)^{2}\right. \\
& \left.+\frac{1}{3}\left(\frac{\theta n+k-\theta}{n(\theta-\tau)}\right)^{3} \frac{1}{1-\left(\frac{\theta * n+k-\theta}{n(\theta-\tau)}\right)^{4}}\right] \\
& +\frac{-n+k-\theta}{n(\theta-\tau)} \arctan \left(\frac{-n+k-\theta}{n(\theta-\tau)}\right)^{2} k \\
& +\frac{k-\theta}{n(\theta-\tau)} \arctan \left(\frac{k-\theta}{n(\theta-\tau)}\right)^{2}(n-k-1) .
\end{aligned}
$$

Since, for any strongly regular graph, we have $q_{0}=0$, then inequality (17) can be rewritten as

$$
\begin{aligned}
0 & \leq \frac{\theta n+k-\theta}{n(\theta-\tau)}\left[\arctan \left(\frac{(\theta n+k-\theta)}{n(\theta-\tau)}\right)^{2}\right. \\
& \left.-\arctan \left(\frac{k-\theta}{n(\theta-\tau)}\right)^{2}\right] \\
& +\frac{1}{3}\left(\frac{\theta n+k-\theta}{n(\theta-\tau)}\right)^{4} \frac{1}{1-\left(\frac{\theta * n+k-\theta}{n(\theta-\tau)}\right)^{4}} \\
& +\frac{n+k-\theta}{n(\theta-\tau)}\left[\arctan \left(\frac{-n+k-\theta}{n(\theta-\tau)}\right)^{2} k\right. \\
& \left.-\arctan \left(\frac{k-\theta}{n(\theta-\tau)}\right)^{2}\right] k .
\end{aligned}
$$

Applying Lagrange's Theorem to the function arctan in the intervals

$$
\left[\left(\frac{k-\theta}{n(\theta-\tau)}\right)^{2},\left(\frac{\theta n+k-\theta}{n(\theta-\tau)}\right)^{2}\right]
$$

$$
\left[\left(\frac{k-\theta}{n(\theta-\tau)}\right)^{2},\left(\frac{n-k+\theta}{n(\theta-\tau)}\right)^{2}\right]
$$

and making some straightforward majorations, one obtains

$$
\begin{aligned}
0 & \leq \frac{\theta n+k-\theta}{n(\theta-\tau)} \frac{1}{1+\left(\frac{k-\theta}{n(\theta-\tau)}\right)^{4}} \frac{\theta}{(\theta-\tau)} \frac{\theta n+2 k-2 \theta}{n(\theta-\tau)} \\
& +\frac{1}{3}\left(\frac{\theta n+k-\theta}{n(\theta-\tau)}\right)^{4} \frac{1}{1-\left(\frac{\theta n+k-\theta}{n(\theta-\tau)}\right)^{4}} \\
& +\frac{-n+k-\theta}{n(\theta-\tau)} \frac{1}{1+\left(\frac{-n+k-\theta}{n(\theta-\tau)}\right)^{4}} \frac{1}{\theta-\tau} \frac{n-2 k+2 \theta}{n(\theta-\tau)} k .
\end{aligned}
$$


Since $k \leq n / 3$ one obtains:

$$
\begin{aligned}
0 \leq & \frac{1+\left(\frac{n-k+\theta}{n(\theta-\tau)}\right)^{4}}{1+\left(\frac{k-\theta}{n(\theta-\tau)}\right)^{4}} \frac{3 \theta+1}{3(\theta-\tau)} \frac{\theta}{\theta-\tau} \frac{3 \theta+2}{3(\theta-\tau)} \\
+ & \left(1+\left(\frac{n-k+\theta}{n(\theta-\tau)}\right)^{4}\right) \frac{1}{3}\left(\frac{3 \theta+1}{3(\theta-\tau)}\right)^{4} \\
& \left.\quad \frac{1}{1-\left(\frac{3 \theta+1}{3(\theta-\tau)}\right)^{4}}\right] \\
+ & \frac{-n+k-\theta}{n(\theta-\tau)} \frac{1}{\theta-\tau} \frac{n-2 k+2 \theta}{n(\theta-\tau)} k .
\end{aligned}
$$

Now, since $\theta<|\tau|-2 / 3$, then we conclude that

$$
\frac{3 \theta+1}{3(\theta-\tau)} \frac{1}{1-\left(\frac{3 \theta+1}{3(\theta-\tau)}\right)^{4}} \leq 1
$$

and

$$
\frac{3 \theta+1}{3(\theta-\tau)} \frac{\theta}{\theta-\tau} \frac{3 \theta+2}{3(\theta-\tau)} \leq\left(\frac{3 \theta+1}{3(\theta-\tau)}\right)^{3},
$$

then we conclude that

$$
\begin{aligned}
& \frac{n-k+\theta}{n(\theta-\tau)} \frac{n-2 * k+\theta}{n(\theta-\tau)} \frac{1}{\theta-\tau)} k \leq\left(\frac{1+\left(\frac{n-k+\theta}{n(\theta-\tau)}\right)^{4}}{1+\left(\frac{(k-\theta)}{n(\theta-\tau)}\right)^{4}}\right. \\
+ & \left.\frac{1+\left(\frac{n-k+\theta}{n(\theta-\tau)}\right)^{4}}{3}\right)\left(\frac{3 \theta+1}{3(\theta-\tau)}\right)^{3}
\end{aligned}
$$

Using the fact that $k<n / 3$ and making an algebraic manipulation of the right member of (18) we come to inequality (19):

$$
\frac{2}{3(\theta-\tau)} \frac{1}{3(\theta-\tau)} \frac{1}{\theta-\tau} k \leq \frac{461}{243}\left(\frac{3 \theta+1)}{3(\theta-\tau)}\right)^{3}
$$

Finally, rewriting inequality (19) and since 461/1458 $<1 / 3$, we obtain

$$
k \leq \frac{1}{3}(3 \theta+1)^{3}
$$

Applying the result presented in Theorem 1 to the complement graph, one obtains the following corollary.

Corollary 1: Let $X$ be a strongly regular graph with parameter set $(n, k, a, c)$ and three distinct eigenvalues, $k, \theta$ and $\tau$. If $k>2 n / 3-1$ and $|\tau|<\theta+4 / 3$, then

$$
n-k-1 \leq \frac{1}{3}(3|\tau|-2)^{3} \text {. }
$$

\section{Numerical Results AND CONCLUSIONS}

In this section we present some experimental results for the feasibility conditions obtained in Section V. We consider the following notation:

$$
q_{\theta k}=\frac{1}{3}(3 \theta+1)^{3}-k
$$

and

$$
q_{\tau k}=\frac{1}{3}(3|\tau|-2)^{3}-(n-k-1)
$$

We also consider the parameter sets $P_{1}=(961,312,41,130)$, $P_{2}=(1225,352,24,132), \quad P_{3}=(1024,385,36,210)$ and the respective complement parameter sets $\bar{P}_{1}=$ $(961,648,465,378), \bar{P}_{2}=(1225,872,651,545)$ and $\bar{P}_{3}=$ $(1024,638,462,290)$.

In Table I we present some experimental results for the inequality of Theorem 1 .

\begin{tabular}{|c||c|c|c|}
\hline Param. & $P_{1}$ & $P_{2}$ & $P_{3}$ \\
\hline \hline$\theta$ & 2.0 & 2.0 & 1.0 \\
\hline$\tau$ & -91.0 & -110.0 & -175.0 \\
\hline$q_{\theta k}$ & -197.7 & -237.7 & -363.7 \\
\hline
\end{tabular}

TABLE I

NUMERICAL RESUlTS FOR $P_{1}=(961,312,41,130)$, $P_{2}=(1225,352,24,132)$ AND $P_{3}=(1024,385,36,210)$.

In Table II we present some experimental results for the inequality of Corollary 1.

\begin{tabular}{|c||c|c|c|}
\hline Param. & $\bar{P}_{1}$ & $\bar{P}_{2}$ & $\bar{P}_{3}$ \\
\hline \hline$\theta$ & 90.0 & 109.0 & 174.0 \\
\hline$\tau$ & -3.0 & -3.0 & -2.0 \\
\hline$q_{\theta k}$ & -197.7 & -237.7 & -363.7 \\
\hline
\end{tabular}

TABLE II

NUMERICAL RESULTS FOR $\bar{P}_{1}=(961,648,465,378)$, $\bar{P}_{2}=(1225,872,651,545)$ AND $\bar{P}_{3}=(1024,638,462,290)$.

Analyzing the results obtained for the inequalities of Theorem 1 and Corollary 1 we can conclude, from inequality (16), that when $k<n / 3$ the value of $k$ cannot be too big compared with the value of $\theta$ and, from inequality (20), when $k>2 n / 3-1$, the value of $n-k-1$ cannot be too big compared with the absolute value of $\tau$.

\section{ACKNOWLEDGMENT}

1) Vasco Moço Mano is supported in part by FEDER funds through COMPETE Operational Programme Factors of Competitiveness ("Programa Operacional Factores de Competitividade") and by Portuguese funds through the Center for Research and Develpment in Mathematics and Applications and the Portuguese Foundation for Science and Technology ("FCT Fundação para a Ciência e a Tecnologia") within project PEest-C/MAT/UI4106/2011 with COMPETE number FCOMP-01-0124-FEDER-022690.

2) Luís Vieira research funded by the European Regional Development Fund through the program COMPETE and by the Portuguese Government through the Center of Mathematics of University of Porto and the Portuguese Foundation for Science and Technology ("FCT - Fundação para a Ciência e a Tecnologia") under the project PEest-C/MAT/UI0144/2011. 


\section{REFERENCES}

[1] R. C. Bose, Strongly regular graphs, partial geometries and partially balanced designs, Pacific J. Math 13, 389-419, 1963.

[2] P. Jordan, J. v. Neuman and E. Wigner, On an algebraic generalization of the quantum mechanical formalism, Annals of Mathematics, Vol 35, 29-64, 1934.

[3] H. Massan and E. Neher, Estimation and testing for lattice conditional independence models on Euclidean Jordan algebras, Ann. Statist., Vol 26, 1051-1082, 1998.

[4] L. Faybusovich, Euclidean Jordan algebras and Interior-point algorithms, J. Positivity, Vol 1, 331-357, 1997.

[5] L. Faybusovich, Linear systems in Jordan algebras and primal-dual interior-point algorithms, Journal of Computational and Applied Mathematics, Vol 86,148-175, 1997.

[6] D. M. Cardoso and L. A. Vieira, Euclidean Jordan Algebras with Strongly Regular Graphs, Journal of Mathematical Sciences, Vol 120, 881-894, 2004.

[7] V. M. Mano, E. A. Martins and L. A. Vieira, Feasibility conditions on the parameters of a strongly regular graph, Electronic Notes in Discrete Mathematics, 38, 607-613, 2011.

[8] V. M. Mano and L. A. Vieira, Admissibility Conditions and Asymptotic Behavior of Srongly Regular Graphs, International Journal of Mathematical Models and Methods in Applied Sciences, Issue 6, Vol 5, 1027-1034, 2011.

[9] C. Godsil and G. Royle, Algebraic Graph Theory, Springer, 2001.

[10] E. Bannai and T. Ito, Algebraic Combinatorics. I. Association Schemes., Benjamin-Cummings, Menlo Park, CA, 1984.

[11] Jr. L. L. Scott, A condition on Higman's parameters, Notices of Amer Math. Soc., 20 A-97, 721-20-45, 1973.

[12] Ph. Delsarte, J. M. Goethals and J. J. Seidel, Bounds for system of lines and Jacobi polynomials, Philips Res. Rep. 30, 91-105, 1975.

[13] A. Neumaier, New inequalities for the parameters of an association scheme, Combinatorics and Graph Theory, Springer Lecture Notes 885, pp. 365-367.

[14] A. E. Brouwer and J. H. van Lint, Strongly regular graphs and partial geometries, Enumeration and Design Academic Press, 1982.

[15] J. Faraut and A. Korányi, Analysis on Symmetric Cones Oxford Science Publication, 1994.

\section{Creative Commons Attribution License 4.0 (Attribution 4.0 International, CC BY 4.0)}

This article is published under the terms of the Creative Commons Attribution License 4.0

https://creativecommons.org/licenses/by/4.0/deed.en US 